\title{
Zellsaftgewinnung, AFS (apparent free space) und Vakuolenkonzentration der osmotisch wichtigsten mineralischen Bestandteile einiger Helgoländer Meeresalgen ${ }^{1}$
}

\author{
HANSWERnER KesSELer \\ Biologische Anstalt Helgoland, Meeresstation, Helgoland
}

\begin{abstract}
Collection of cell sap, AFS (apparent free space) and vacuole concentration of the osmotically most important mineral components of some Helgoland marine algae. Samples of cell sap in amounts suitable for chemical analysis of mineral compounds were obtained by high speed centrifugation of several frost-killed $\left(-20^{\circ} \mathrm{C}\right)$ Helgoland algae under paraffin oil. In order to calculate the vacuole concentration of the mineral salts determined in these liquids, the extravacuolar solving space of the algae was measured as Lithium apparent free space (Li-AFS) after rinsing the algae for several minutes in balanced $\mathrm{LiCl}$ solutions. The Li-AFS was calculated from the results gained by flamephotometric determination of the Li-concentrations in the sap and in the bathing fluid. To avoid errors caused by interaction of Donnan-effects, the Li-AFS of Chaetomorpha linum kept in diluted sea water of different concentrations was measured. By plotting the results against the medium concentration, evidence was obtained to show that in $100 \% \mathrm{Li}$-solution (corresponding osmotically to natural sea water of about $30 \%$ ), the Donnan effect was negligible. In the more diluted solutions, however, higher Li-AFS values were obtained. This would indicate that the differences in the distribution of $\mathrm{Li}$-cations between medium and AFS, which are effected by the negative charges of indiffusible anions in the cytoplasm and of fixed acidic groups (e. g. $\mathrm{R}-\mathrm{COO}^{-}$) of phycocolloids in the wall material, will cause considerable errors if Li-concentrations are too low. Taking these experiences into account, AFS-values of several algae from Helgoland were measured by the same technique and the vacuole concentrations of the analysed mineral compounds calculated.
\end{abstract}

\section{EINLEITUNG}

Die Gewinnung größerer, für chemisch-analytische Untersuchungen ausreichender Zellsaftmengen aus Meeresalgen stieß bisher - abgesehen von ganz wenigen Ausnahmefällen - auf unüberwindliche Schwierigkeiten. Bei den Ausnahmen handelte es sich um einige extrem großzellige Formen, von denen sich der Zellsaft verhältnismäßig leicht in genügender Quantität gewinnen ließ (Literatur bei ColLANDER 1930, 1939). Die an diesen Objekten gewonnenen Ergebnisse durften jedoch nicht ohne weiteres verall-

${ }^{1}$ Herrn Professor Dr. Adolf Bückmann zum 65. Geburtstag in Verehrung gewidmet. 
gemeinert werden. Gerade ihre morphologische Sonderstellung ließ vermuten, daß sie auch in bezug auf ihren Stoffwechsel von der "Norm" abweichen würden, denn Struktur und Funktion sind untrennbar miteinander verknüpt und nur verschiedene Aspekte ein und derselben Gegebenheit: des lebendigen Organismus.

Inzwischen ist es gelungen, eine Methodik zu entwickeln, mit deren Hilfe es möglich ist, auch von kleinzelligen Meeresalgen Saft in Mengen zu gewinnen, welche die Durchführung von chemischen Analysen mit den üblichen Makrobestimmungsverfahren gestatten. Die Anwendung des neuen Verfahrens (siehe unter Methodik) hat den einzigen Nachteil, daß es dabei zu einer Vermischung des Vakuolensaftes mit dem Haft- bzw. Imbibitionswasser der Zellwände, der Interzellularräume und des Protoplasmas kommt. Zur Eliminierung des dadurch bedingten Verdünnungsfehlers mußs also diese Wassermenge oder, was praktisch das gleiche bedeutet, die Größe des extravakuolaren Lösungsraumes bekannt sein. Sie läßt sich unter Berücksichtigung der besonderen, objektbedingten Gegebenheiten als "apparent free space“ (AFS) bestimmen.

\section{ALLGEMEINES}

Seiner ursprünglichen, von Hope \& STEveNs (1952) gegebenen Definition gemäß sollte der AFS einer Zelle derjenige Raum sein, in den eine gegebene Außenlösung und zwar sowohl die Molekeln des Lösungsmittels als auch die der gelösten Substanz passiv und reversibel durch Diffusion eindringt. Das Hauptanliegen der auf dieser Begriffsbestimmung basierenden Untersuchungsmethoden war zunächst, zu ergründen, wo das entscheidende Hindernis für die freie Diffusion gelöster Substanzen zu suchen sei: im Plasmalemma oder im Tonoplasten.

Eingehende Untersuchungen dieser Frage (Literatur bei Collander 1956, KraMER 1956) führten zu dem Ergebnis, daß für den AFS ein und desselben Objektes, je nach Art und Konzentration der verwendeten Testsubstanz, unterschiedliche Werte gemessen wurden (Hope 1953, ButLER 1953). Bei Verwendung relativ großmolekularer Anelektroly te (z. B. Rohrzucker) wurde in exster Linie der extraplasmatische lösende Raum der Zellwände und Interzellularräume als AFS bestimmt. Demgegenüber ließ die deutliche Konzentrationsabhängigkeit der mit Hilfe von Elektrolyten ermittelten AFS-Werte die Auswirkungen Donnanscher Ionenverteilungen sichtbar werden. In diesen Fällen war offenbar auch der Zytoplasmaraum - oder zumindest ein Teil desselben - miterfaßt worden.

Dieser Umstand ließ die Bestimmung des hier interessierenden extravakuolaren Lösungsraumes mit Hilfe eines geeigneten Elektrolyten unter Beachtung der durch den DONNAN-Effekt bedingten Fehlermöglichkeiten aussichtsreich erscheinen. Die Verwendung eines Elektrolyten zur Bestimmung des AFS von Meeresalgen war schon deshalb der Benutzung von Anelektrolyten vorzuziehen, weil diese Pflanzengruppe ja auch unter natürlichen Bedingungen in einem Medium hoher Elektrolytkonzentration lebt. Der als Testsubstanz zu verwendende Elektrolyt mußte jedoch folgende Bedingungen erfüllen: 1. Er mußte rasch in den gesamten extravakuolaren Lösungsraum, vor allem auch in das Zytoplasma, eindringen können, ohne gleichzeitig auch die Vakuolen zu überschwemmen. 2. Zur Ausschaltung des DonNan-Effektes mußte er in so hoher 
Konzentration in die extravakuolaren Zellbereiche gelangen können, daß demgegenüber die Konzentration der indiffusiblen Ionen des Protoplasmas bzw. der fixierten Ladungen des Zellwandmaterials nicht mehr ins Gewicht fiel. 3. Er mußte mit den üblichen Nachweismethoden gut erfaßbar und quantitativ bestimmbar sein.

Bei Erfüllung dieser Voraussetzungen läßt sich aus der gemessenen Elektrolytkonzentration der gewonnenen Säfte $\left(\left[\mathrm{El}_{\mathrm{s}}\right]\right.$ ) bei bekannter Mediumkonzentration ( $\left.\left[\mathrm{El} \mathrm{l}_{\mathrm{m}}\right]\right)$ der als AFS definierte extravakuolare Lösungsraum, der im vorliegenden Falle natürlich auch noch die besonders bei feinfädigen Meeresalgen nicht unbeträchtliche Menge des Haftwassers mitumfaßt, in einfacher Weise berechnen. Da sich nämlich der AFS zum Gesamtzellvolumen $\left(V_{z}\right)$ offenbar ebenso verhält, wie die Elektrolytkonzentration des Saftes zu derjenigen des Außenmediums, so folgt:

$$
\frac{\mathrm{AFS}}{\mathrm{V}_{\mathrm{z}}}=\frac{\left[\mathrm{E} l_{\mathrm{s}}\right]}{\left[\mathrm{E} l_{\mathrm{m}}\right]} ; \mathrm{AFS}=\mathrm{V}_{\mathrm{z}} \frac{\left[\mathrm{E} l_{\mathrm{s}}\right]}{\left[\mathrm{E} l_{\mathrm{m}}\right]}
$$

Setzt man in dieser Gleichung $V_{z}=100$, so erhält man den AFS in Prozenten des Zellyolumens. Das Vakuolenvolumen ist dann

$$
\mathrm{V}_{\mathrm{vac}} \%=100-\operatorname{AFS} \%
$$

und der Korrektionsfaktor $f$ zur Umrechnung der Analysenergebnisse auf Vakuolenkonzentration

$$
f=\frac{100}{V_{\text {vac }} \%}
$$

Diese Gleichungen gelten allerdings nur dann in Strenge, wenn die in den Zellsäften nachgewiesenen Substanzen - mit Ausnahme des Testelektrolyten natürlich - tatsächlich nur aus dem Vakuolenraum stammen. Bei zellsaftreichen Meeresalgen ist diese Bedingung, wie noch zu zeigen sein wird, nach der Elektrolytvorbehandlung weitgehend erfüllt.

Ein Testelektrolyt, der die drei vorgenannten Bedingungen in nahezu idealer Weise erfüllte, wurde im Lithiumchlorid gefunden. Wie EppLEY (1959) an Porphyra perforata zeigen konnte, dringt das Li-Ion gut in das Protoplasma ein. In der Vakuole ist es dagegen erst nach längeren Versuchszeiten nachzuweisen, wie schon Cooper et al. (1929) an Valonia macrophysa und COLLANDER (1939) an Chara ceratophylla fanden. Wegen seiner nahen Verwandtschaft zum $\mathrm{Na}$ läßt es sich gut als Hauptkation für seewasserisotonische Lösungen verwenden und damit in der zur Ausschaltung des Donnan-Effektes erwünschten hohen Konzentration zur Anwendung bringen. Darüber hinaus ist es flammenphotometrisch gut nachweisbar und damit quantitativ leicht $\mathrm{zu}$ bestimmen.

\section{METHODIK}

Das für die Untersuchungen verwandte Material wurde bis zum Versuchsbeginn in fließendem Seewasser bei zwölfstündiger künstlicher Beleuchtung durch Fluoreszenzlampen aufbewahrt. Materialportionen von 10 bis $20 \mathrm{~g}$ Frischgewicht wurden nach Entfernung der Hauptmenge des Haftwassers durch Schleudern in einem Tuch in einer 
seewasserisotonischen (气 $30 \%$ Salzgehalt), balancierten LiCl-Lösung folgender Zusammensetzung abgespült:

$$
\begin{aligned}
& \mathrm{LiCl} \quad=20 \mathrm{~g} / \mathrm{l}\left(\hat{=} 4,71 \cdot 10^{-1} \mathrm{~mol}\right) \\
& \mathrm{SrCl}_{2} \cdot 6 \mathrm{H}_{2} \mathrm{O}=5 \mathrm{~g} / \mathrm{l} \text { (全 } 1,87 \cdot 10^{-2} \mathrm{~mol} \text { ) } \\
& \mathrm{NaHCO}=200 \mathrm{mg} / \mathrm{l}\left(\triangleq 2,38 \cdot 10^{-3} \mathrm{~mol}\right. \text { ) }
\end{aligned}
$$

Die Spülzeiten betrugen, je nach der Beschaffenheit der zu untersuchenden Algen, 1 bis 5 Minuten. Nach dem Spülen wurde zunächst wieder das Haftwasser in der beschriebenen Weise entfernt. Danach wurden gleiche Materialportionen eingewogen und in $150 \mathrm{ml}$-Zentrifugengläser übertragen, die mit einem Siebeinsatz aus Kunststoff versehen waren (Abb. 1). Die so vorbereiteten Gläser wurden mit Paraffinöl gefüllt, gut verschlossen und zum Abtöten der Algen über Nacht in einer Tiefkühltruhe bei $-20^{\circ} \mathrm{C}$ aufbewahrt. In einigen Fällen wurden die Algen auch durch Ubergießen mit heißem Paraffinöl $\left(95^{\circ} \mathrm{C}\right)$ abgetötet und dann ebenfalls bis zur Weiterverarbeitung kühl gestellt.

Zur Gewinnung des Zellsaftes wurden die Proben aufgetaut und anschließend auf einer Kühlzentrifuge (CHRIST „Universal Junior III KS“) bei $+5^{\circ} \mathrm{C}$ und 4500 U.p. M. (스 $4800 \mathrm{~g}_{\max }$ ) eine Stunde lang zentrifugiert. Auf diese Weise wurde von allen bisher untersuchten Algen klarer, durch die wasserlöslichen Chromatophorenfarbstoffe aller-

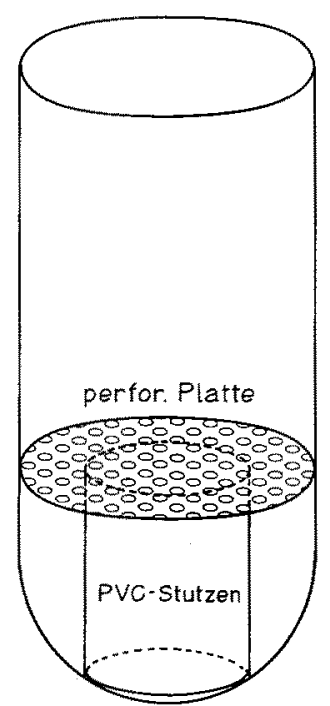

Abb. 1: Zentrifugenglas mit Siebeinsatz zur Zellsaftgewinnung

dings leicht braun bis rot gefärbter Zellsaft gewronnen, der sich unterhalb des Siebeinsatzes sammelte. Nach Entfernung des Siebeinsatzes mit dem Rückstand des Materials wurde der Zellsaft in Zelluloseröhrchen dekantiert und auf der Ultrazentrifuge (BECKMAN „Spinco L“) bei $+5^{\circ} \mathrm{C}$ und 25000 U.p.M. (스 $\left.90000 \mathrm{~g}_{\max }\right)^{1 / 2}$ Stunde lang nachzentrifugiert. Von den so behandelten Proben wurden aliquote Teile abpipettiert und je nach Konzentration der nachzuweisenden Ionen mit destilliertem Wasser ver- 
dünnt. In diesen Lösungen wurde die Bestimmung der wichtigsten anorganischen Ionen vorgenommen.

$\mathrm{Li}, \mathrm{Na}^{*}$ und $\mathrm{K}^{*}$ wurden flammenphotometrisch (BECKMAN Spektralphotometer Modell DU mit Flammenzusatz) mit Hilfe verschiedener, auf die Ionenverhältnisse der einzelnen Algengruppen abgestimmter Eichreihen gemessen.

$\mathrm{Ca}$ " plus $\mathrm{Mg}$ " wurden komplexometrisch mit AeDTE und Merck-Indikatorpuffertabletten bei $\mathrm{p}_{\text {II }} 10$ bestimmt, dann $\mathrm{Ca}$ " allein ebenfalls mit AeDTE unter Verwendung von Calconcarbonsäure als Indikator bei $\mathrm{p}_{\mathfrak{H}} 13$.

$\mathrm{Cl}^{\prime}$ wurde argentometrisch mit $\mathrm{n} / 20 \mathrm{AgNO}_{3}$ nach $\mathrm{MoHR}$ titriert, $\mathrm{SO}_{4}{ }^{\prime \prime}$ nach Fällung als $\mathrm{BaSO}_{4}$ gravimetrisch ermittelt und $\mathrm{PO}_{4}{ }^{\prime \prime \prime}$ kolorimetrisch mit Hilfe der Molybdänblaureaktion unter Verwendung eines ZEISS „Elko II" gemessen.

\section{ERGEBNISSE}

Zunächst wurde an Chatomorpha linum der Einfluß des Donnan-Effektes auf die Größe des Li-AFS studiert. Dazu wurden Materialproben von $10 \mathrm{~g}$ Frischgewicht in Kunststoffschalen mit 11 Seewasser verschiedener Konzentrationen (100\%, 80\%, $60 \%, 40 \%, 20 \% ; 100 \%$ 을 $30 \%$ Salzgehalt) unter Zusatz von je $10 \mathrm{ml}$ Erdabkochung, $1 \mathrm{ml} \mathrm{NaNO}$-Lösung (10\%) und $1 \mathrm{ml} \mathrm{NaH} 2 \mathrm{PO}_{4} \cdot 12 \mathrm{H}_{2} \mathrm{O}$-Lösung (2\%) übertragen. In diesen Lösungen wurden die Algen bei täglichem Wasserwechsel 3 Tage lang zur Anpassung belassen. Nach dieser Zeit wurden sie in der beschriebenen Weise verarbeitet. Zur Spülung ( 2 Minuten) wurden LiCl-Lösungen entsprechender Konzentrationen verwandt. Je $5 \mathrm{~g}$ Frischmaterial der einzelnen Verdünnungsstufen wurden bei $-20^{\circ} \mathrm{C}$ abgetötet, die restlichen $5 \mathrm{~g}$ bei $+95^{\circ} \mathrm{C}$. Im übrigen wurden die Proben gleich behandelt.

Die Ergebnisse beider Versuchsserien sind in Tabelle 1 wiedergegeben und in Abbildung 2 graphisch dargestellt. Sie zeigen, daß bei Anwendung von LiCl-Konzen-

\section{Tabelle 1}

Li-AFS von Chaetomorpha linum in Abhängigkeit von der Li-Konzentration im Außenmedium. SW: Salzgehalt des Seewassers, in welchem sich die Algen vor Versuchsbeginn 3 Tage lang bei täglichem Wasserwechsel befunden hatten

\begin{tabular}{|c|c|c|c|c|}
\hline \multicolumn{2}{|c|}{$\begin{array}{l}\text { Li-Konzentration } \\
\text { relativ } \quad \mathrm{mol} / 1\end{array}$} & SW & $\mathrm{AFS} \%$ & Vorbehandlung \\
\hline $\begin{array}{r}100 \% \\
80 \% \\
60 \% \\
40 \% \\
20 \% \\
\end{array}$ & $\begin{array}{l}0,475 \\
0,380 \\
0,285 \\
0,190 \\
0,095\end{array}$ & $\begin{array}{l}30 \% \\
24 \% \\
18 \% \\
12 \% \\
6 \%\end{array}$ & $\begin{array}{l}21,1 \\
22,2 \\
25,0 \\
26,0 \\
36,7\end{array}$ & $\begin{array}{l}\text { alle Proben } 2 \text { Min. in } \\
\text { LiCl-Lösungen gespült } \\
\text { Material bei }-20^{\circ} \mathrm{C} \\
\text { abgetötet }\end{array}$ \\
\hline $\begin{array}{r}100 \% \\
80 \% \\
60 \% \\
40 \% \\
20 \%\end{array}$ & $\begin{array}{l}0,475 \\
0,380 \\
0,285 \\
0,190 \\
0,095\end{array}$ & $\begin{array}{l}30 \% 0 \\
24 \% \\
18 \% \\
12 \% 0 \\
6 \%\end{array}$ & $\begin{array}{c}21,3 \\
(27,2) \\
23,4 \\
24,7 \\
35,7\end{array}$ & $\begin{array}{l}\text { alle Proben } 2 \text { Min. in } \\
\text { LiCl-Lösungen gespült } \\
\text { Material bei }-95^{\circ} \mathrm{C} \\
\text { abgetöret }\end{array}$ \\
\hline
\end{tabular}


trationen unterhalb etwa $0,4 \mathrm{~mol}(\hat{=} \mathrm{ca} .80 \%$ relative Li-Konzentration, $\triangleq \mathrm{ca} .25 \%$ Seewasser) durch den Einfluß des DonNan-Effektes bereits merkliche, reproduzierbare Fehler hinsichtlich der Größe des Li-AFS verursacht werden. Oberhalb der gewählten $100 \%$-Konzentration bleiben die AFS-Werte dagegen praktisch konstant, ein Beweis dafür, daß die durch den Donnan-Effekt verursachten Li-Konzentrationsunterschiede zwischen AFS und Außenmedium nicht mehr ins Gewicht fallen. Unter diesen Bedingungen herrscht also nicht nur Aktivitäts- sondern praktisch auch Konzentrationsgleichgewicht $z$ wischen beiden Räumen.

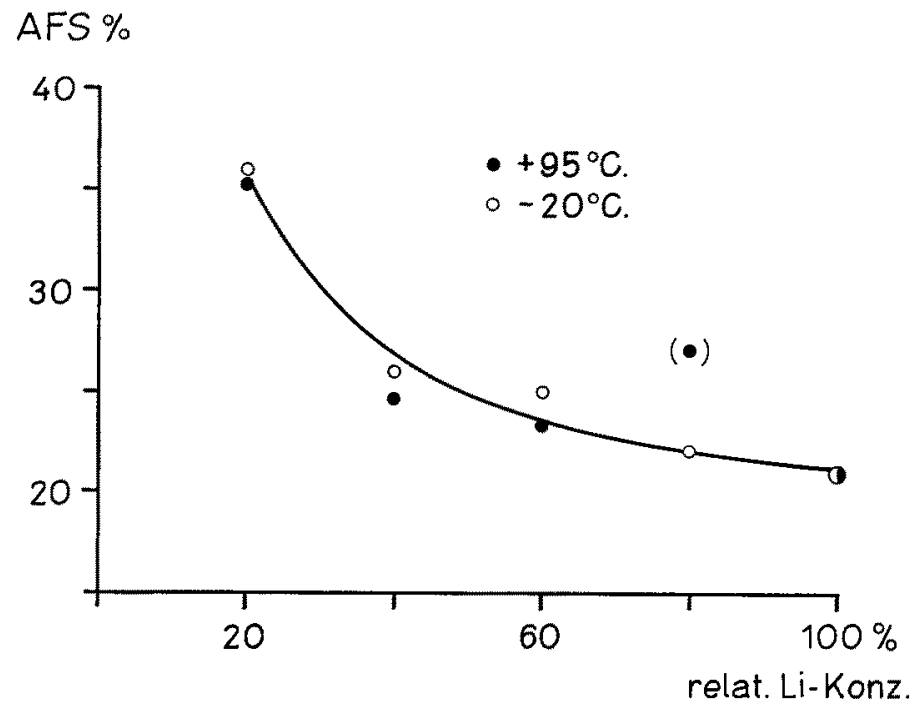

Abb. 2: Scheinbare Änderung der Größe des Lithium-AFS („apparent free space“) von Chaetomorpha linum als Folge des Donnan-Effektes

Ober die Reproduzierbarkeit der Li-AFS-Werte gleicher Materialproben von Chaetomorpha linum bzw. über die möglichen Unterschiede bei gleichartigem Material, das zu verschiedenen Zeiten gesammelt wurde, unterrichtet Tabelle 2:

Tabelle 2

Li-AFS-Werte verschiedener Materialproben von Chaetomorpha linum

\begin{tabular}{|llll|}
\hline Sammeldatum & Herkunft & AFS \% & Vorbehandlung \\
\hline 21. 7. 1964 & List/Sylt & 21,5 & \\
& List/Sylt & 21,1 & Materialproben 2 Min. in \\
& List/Sylt & 21,6 & $100 \%$-LiCl-Lösung ge- \\
\hline \multirow{2}{*}{27.8 .1964} & List/Sylt & 25,3 & spült und bei $-20^{\circ} \mathrm{C}$ ab- \\
& List/Sylt & 25,3 & getötet \\
& List/Sylt & 25,9 & \\
\hline
\end{tabular}

In Tabelle 3 sind die Li-AFS-Werte einiger Helgoländer Meeresalgen zusammengestellt. Sie zeigen, daß auch die Methode der Zellsaftgewinnung für die Bestimmung 
Tabelle 3

AFS (Apparent Free Space) einiger Helgoländer Meeresalgen aus Seewasser von $30 \%$ Salzgehalt

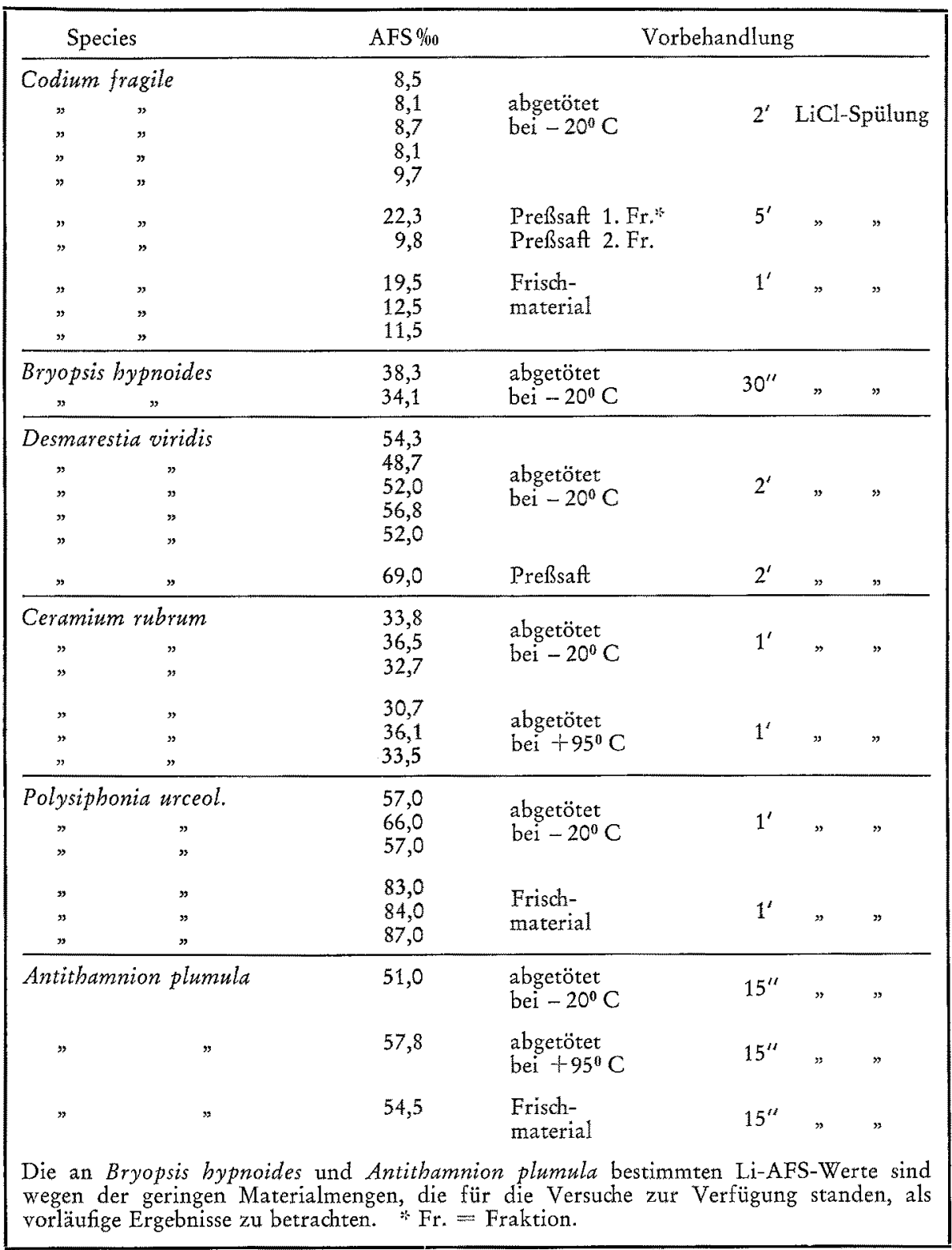

des AFS von Bedeutung ist. Preßsäfte und Säfte von Algen, die ohne vorherige $\mathrm{Ab}$ tötung in frischem Zustand zentrifugiert und verarbeitet wurden, führen zu unbrauchbaren Ergebnissen. Offenbar konnte in diesen Fällen wegen der Ultrafilterwirkung 
des noch lebenden Protoplasmas nur verdünnter Zellsaft gewonnen werden, in welchem das aus dem extravakuolaren Raum stammende Lithium in relativ zu hoher Konzentration vorlag. Infolgedessen ergab die Berechnung zu hohe AFS-Werte.

Tabelle 4 bringt schließlich noch die an Zellsäften einiger Helgoländer Meeresalgen ermittelten Analysenergebnisse. Sie wurden unter Berücksichtigung der Li-AFSWerte auf das Vakuolenvolumen umgerechnet. Schon diese wenigen Daten zeigen, daß den Mineralstoffwechselprozessen der verschiedenen Vertreter dieser interessanten Pflanzengruppe trotz weitgehender Gleich heit ihrer Lebensbedingungen offenbar recht unterschiedliche Mechanismen für den Ionentransport und die Ionenregulation zugrunde liegen. Unter diesen Umständen dürfte es kaum möglich sein, ein allgemein gültiges Schema zu ersinnen, das allen Forderungen, die sich aus diesem bunten Bild ergeben, in zufriedenstellender Weise Rechnung trägt.

\section{Tabelle 4}

Konzentrationen und Ionenrelationen der wichtigsten anorganischen Komponenten des Seewassers im Zellsaft einiger Helgoländer Meeresalgen aus $30 \%$

\begin{tabular}{|c|c|c|c|c|c|c|c|}
\hline \multicolumn{2}{|c|}{ Untersuchte Arten } & $\begin{array}{l}\text { Chaeto- } \\
\text { morpba } \\
\text { linum }\end{array}$ & $\begin{array}{l}\text { Codium } \\
\text { fragile }\end{array}$ & $\begin{array}{c}\text { Bryopsis } \\
\text { bypnoides }\end{array}$ & $\begin{array}{c}\text { Des- } \\
\text { marestio } \\
\text { viridis }\end{array}$ & $\begin{array}{l}\text { Cera- } \\
\text { mium } \\
\text { rubrum }\end{array}$ & $\begin{array}{c}\text { Poly- } \\
\text { sipbonia } \\
\text { urceolata }\end{array}$ \\
\hline \multicolumn{2}{|c|}{ Anzahl der Proben } & 10 & 5 & 2 & 5 & 6 & 3 \\
\hline \multirow[t]{2}{*}{ 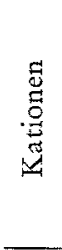 } & $\begin{array}{l}\mathrm{H}^{*} \\
\mathrm{Na} . \\
\mathrm{K} \cdot \\
\mathrm{Mg} . \\
\mathrm{Ca} .\end{array}$ & $\begin{array}{l}\overline{0,044} \\
0,743 \\
0,033 \\
0,008\end{array}$ & $\begin{array}{c}-\overline{0} 483 \\
0,015 \\
0,081 \\
0,068\end{array}$ & $\begin{array}{l}- \\
0,345 \\
0,280 \\
0,004 \\
0,002\end{array}$ & $\begin{array}{l}0,383 \\
0,111 \\
0,279 \\
0,099 \\
0,057\end{array}$ & $\begin{array}{l}- \\
0,031 \\
0,515 \\
0,150 \\
0,015\end{array}$ & $\begin{array}{c}-\overline{0} \\
0,096 \\
0,644 \\
0,095 \\
0,058\end{array}$ \\
\hline & $\begin{array}{l}\sum \text { Kationen } \\
(\mathrm{Val} / \mathrm{l})\end{array}$ & 0,828 & 0,647 & 0,631 & 0,929 & 0,711 & 0,893 \\
\hline \multirow{2}{*}{ 离 } & $\begin{array}{l}\text { E Anionen } \\
(\mathrm{Val} / \mathrm{l})\end{array}$ & 0,815 & 0,653 & 0,738 & 0,968 & 0,667 & 0,784 \\
\hline & $\begin{array}{l}\mathrm{Cl}^{\prime} \\
\mathrm{SO}_{4}{ }^{\prime \prime} \\
\mathrm{PO}_{4}^{\prime \prime \prime}\end{array}$ & $\begin{array}{l}0,762 \\
0,045 \\
0,008\end{array}$ & $\begin{array}{l}0,551 \\
0,100 \\
0,002\end{array}$ & $\begin{array}{l}0,705 \\
0,028 \\
0,005\end{array}$ & $\begin{array}{l}0,089 \\
0,872 \\
0,007\end{array}$ & $\begin{array}{l}0,575 \\
0,088 \\
0,004\end{array}$ & $\begin{array}{l}0,583 \\
0,198 \\
0,003\end{array}$ \\
\hline \multirow{5}{*}{ 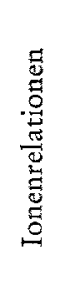 } & $\begin{array}{l}\Sigma \text { Kationen } \\
\Sigma \text { Anionen }\end{array}$ & 1,016 & 0,991 & 0,855 & 0,960 & 1,065 & 1,139 \\
\hline & $\mathrm{Na}_{\mathrm{i}}: \mathrm{Na}_{\mathrm{e}}$ & 0,107 & 1,180 & 0,842 & 0,270 & 0,076 & 0,234 \\
\hline & $\mathrm{K}_{\mathrm{i}}: \mathrm{K}_{\mathrm{e}}$ & 84,7 & 1,71 & 31,9 & 31,75 & 58,6 & 73,3 \\
\hline & $\mathrm{Cl}_{\mathrm{i}}: \mathrm{Cl}_{\mathrm{e}}$ & 1,575 & 1,14 & 1,46 & 0,184 & 1,19 & 1,21 \\
\hline & $\mathrm{SO}_{4 \mathrm{i}}: \mathrm{SO}_{4 \mathrm{e}}$ & 0,985 & 2,18 & 0,61 & 19,2 & 1,925 & 4,32 \\
\hline \multicolumn{8}{|c|}{$\begin{array}{l}\text { Die geringfügigen Abweichungen des Verhältnisses Kationen : Anionen rom Quotienten } 1 \\
\text { sprechen dafür, daß durch die Analysen die osmotisch wichtigsten Ionen des Zellsaftes } \\
\text { nahezu quantitativ erfaßt wurden. Die etwas größeren Differenzen bei Bryopsis bypnoides } \\
\text { und Polysiphonia urceolata dürften in erster Linie auf die geringe Anzahl der bisher } \\
\text { untersuchten Proben zurickzuführen sein. Die Indizes i (= intern) bzw. e (= extern) } \\
\text { bezeichnen die Konzentrationen im Innen- bzw. im Außenmedium. }\end{array}$} \\
\hline
\end{tabular}




\section{DISKUSSION DER ERGEBNISSE}

Bisher wurden erst an wenigen Meeresalgen AFS-Bestimmungen durchgeführt (Literatur bei EPPLEY 1962). Sie hatten in erster Linie die Erfassung der extraplasmatischen Zellräume zum Ziel. Dazu arbeitete man zumeist mit Rohrzuckerlösungen, einer Testsubstanz also, für die das Plasmalemma allgemein als undurchlässig gilt (Scott \& Hayward 1954, Eppley \& Blinks 1957, Follmann \& Follmann-Schrag 1959). Die zuletzt genannten Autoren fanden jedoch, daß diese Methode offenbar nicht in allen Fällen sichere Aussagen über die Größe des AFS von Meeresalgen gestattet. An Cladophora gracilis bestimmten sie nämlich einen AFS-Wert von 21,4\%, während die Berechnung der "Zellwandmasse" nach mikrophotographischen Schichtenaufnahmen nur 14\% ergab. Eine Beeinflussung des Quellungszustandes der Zellwände, wie sie unter anderem von KoTTE (1914) und HofrMANN (1932) beim Arbeiten mit Rohrzucker- und Rohrzucker-Seewasser-Lösungen beobachtet worden war, konnten die Autoren bei ihrem Objekt nicht feststellen. Sie ziehen daraus den Schluß, daß in den AFS auch Partien des Protoplasmas einbezogen sind. Vielleicht handelte es sich hierbei aber weniger um ein Eindringen des Zuckers in das Protoplasma - das Gleichgewicht $z$ wischen AFS und Außenmedium hatte sich nämlich bereits nach 2 Minuten eingestelltals vielmehr um eine reversible Adsorption der polar gebauten Rohrzuckermoleküle an polare Gruppen des Zellwandmaterials und des Plasmalemmas.

An Porpbyra perforata wurde der AFS sowohl mit Rohrzudker (Eppley 1958, Tab. II) als auch mit radioaktivem Sulfat (Eppley \& Cyrus 1960) bestimmt. Im letzteren Falle beobachteten die Autoren in scheinbarem Widerspruch $z u$ unseren, an Chaetomorpha linum gewonnenen Ergebnissen eine Zunahme des AFS mit steigender Seewasserkonzentration. Die von den Autoren selbst gegebene Deutung dieses Befundes, der AFS der Versuchspflanzen aus den Ansätzen mit verdünntem Seewasser sei infolge der Quellung des Materials in diesen Medien kleiner geworden, scheint mir jedoch nicht vertretbar zu sein. Eine sichtbare Quellung hat nämlich immer eine Volumzunahme zur Folge und dürfte daher kaum auf Kosten des AFS erfolgen. Wäre das der Fall, dann hätte auch bei unserem Objekt in den verdünnten Seewasserlösungen eine Abnahme des AFS zu beobachten sein müssen. Statt dessen wurden größere Werte gemessen.

Die einzige Erklärung, die diesen scheinbaren Widersprtich zu beseitigen vermag, dürfte auch in diesem Falle in einer sichtbaren Auswirkung des DonnaN-Effektes zu suchen sein. $\mathrm{Da}$ die Autoren jedoch die Konzentration eines Anions als Grundlage für die Berechnung der AFS-Werte benutzten, mußten sie im Gegensatz zu unserem Befund mit abnehmender Mediumkonzentration kleinere AFS-Werte messen. In verdünnten Lösungen wird nämlich die Konzentration der Anionen wegen der negativen Ladungen der Protoplasma- und Zellwandkolloide durch den Donnan..Effekt negativ beeinflußt.

Der maximale AFS-Wert, den die Autoren mit der Radio-Sulfatmethode ermittelten, stimmt übrigens recht gut mit dem durch die Rohrzuckermethode gemessenen Wert überein, ein Beweis dafür, daß auch das $\mathrm{SO}_{4}$-Ion offenbar nur in den extraplasmatischen Raum genügend rasch einzudringen vermag.

Bei der Umrechnung der chemischen Analysenergebnisse wurde, wie bereits er- 
wähnt, vorausgesetzt, daß die nachgewiesenen Substanzen in der Hauptsache aus den Vakuolen stammten. Für die osmotisch wichtigsten Ionen $\mathrm{K}, \mathrm{Na}^{\circ}$ und $\mathrm{Cl}^{\prime}$ dürfte diese Annahme - vor allem auch im Hinblick auf die Tatsache, daß es sich bei den untersuchten Formen um zellsaftreiche Algen handelte - aus folgenden Grïnden gerechtfertigt sein: Abgesehen von der unbedeutenden Menge an Bicarbonat enthielt die Li-Testlösung nur $\mathrm{Cl}^{\prime}$ als Anion. Nadh der Spülung des Versuchsmaterials war demnach auch im extravakuolaren Lösungsraum der Algen praktisch nur $\mathrm{Cl}^{\prime}$ als frei bewegliches Anion vorhanden. Dieses $\mathrm{Cl}^{\prime}$ mußte natürlich zusammen mit $\mathrm{Li}$ als Gegenion in den zentrifugierten Säften enthalten sein. Zur Errechnung des Cl'-Gehaltes der Vakuolen brauchte daher nur das $\mathrm{Cl}^{\prime}$-Aquivalent des flammenphotometrisch bestimmten Lithiums von den Titrationsergebnissen der Säfte subtrahiert zu werden.

Alle übrigen Ionen, die vor der Spülung des Materials in dessen AFS in frei beweglicher Form vorhanden gewesen waren, mußten durch das Auswaschen daraus entfernt beziehungsweise durch $\mathrm{Li}$ und $\mathrm{Cl}^{\prime}$ substituiert worden sein. Tatsächlich betrug der $\mathrm{SO}_{4}$-Gehalt des Saftes von Chaetomorpha linum nach der Spülung nur noch etwa $35 \%$ seiner Konzentration im Saft von ungespiiltem Material. Der Phosphatspiegel, der osmotisch jedoch bedeutungslos ist, blieb dagegen nahezu konstant. Auch für das $\mathrm{Na}$ wurde bei LiCl-behandeltem Material ein um etwa $65 \%$ niedrigerer Wert gemessen. Die Summe aus $\mathrm{Na}{ }^{*}$ plus $\mathrm{Li}$-Konzentration stimmte dagegen recht gut mit der $\mathrm{Na}$-Konzentration des Saftes ungespïlter Algen überein. Demnach wurde der größte Teil des extravakuolaren Natriums - wenn nicht gar alles - durch $\mathrm{Li}$ substituiert. Eine Konzentrationsabnahme durch die LiCl-Spülung wurde auch beim $\mathrm{Mg}$ “ (um etwa $20 \%$ ) und beim $\mathrm{Ca}$ (um etwa 50\%) festgestellt. Beide Kationen sind allerdings osmotisch kaum von Bedeutung. Es ist daher in diesem Zusammenhang unwesentlich, ob sie aus dem AFS oder aus dem Zellsaftraum stammten.

Das einzige Ion, dessen Konzentration im Saft yon gespiiltem wie von ungespuiltem Material praktisch unverändert blieb, war das K: Demnach kann seine A k tivität im AFS der untersuchten Algen nicht groß gewesen sein. Auf Grund der Untersuchungsbefunde vieler Autoren (Dainty 1960, Diamond \& Solomon 1959, Eppley 1958, 1959, Eppley \& Cyrus 1960, Holm-Jensen et al. 1944, MacRobbie 1962, 1964, MacRobite \& Dainty 1958) muß jedoch geschlossen werden, daß es im Zytoplasma dennoch in recht erheblicher Konzentration vorliegt. Dieser Sachverhalt läßt sich nur durch die Annahme erklären, daß das extravakuolare $\mathrm{K}$ an Bestandteile des Protoplasmas gebunden oder adsorbiert ist. $\mathrm{Da}$ das $\mathrm{K}^{\prime}$ in den Vakuolen ebenfalls in hoher Konzentration - allerdings frei beweglich - vorliegt, dürfte das extravakuolar fixierte Kalium mit Rücksicht auf die schonende Abtötung des Materials durch Einfrieren bei der Zellsaftgewinnung kaum aus dem Protoplasma ausgewaschen worden sein. Auf Grund dieser Ubberlegungen darf, vor allem auch im Hinblick auf den Zellsaftreichtum der untersuchten Algen, geschlossen werden, daß die mitgeteilten Analysenergebnisse weitgehend den Ionenverhältnissen der osmotisch wichtigsten anorganischen Substanzen im Zellsaft entsprechen. 


\section{ZUSAMMENFASSUNG}

1. Es wird ein einfaches Verfahren zur Gewinnung größerer, für chemisch-analytische Untersuchungen ausreichender Zellsaftmengen auch von kleinzelligen Meeresalgen beschrieben.

2. Eine neue Methode zur Bestimmung des AFS von Meeresalgen mit Hilfe seewasserisotonischer LiCl-Lösungen wird am Beispiel von Chaetomorpha linum kritisch untersucht.

3. Unter Berücksichtigung der an einigen Helgoländer Meeresalgen bestimmten Li-AFS-Werte werden aus den Ergebnissen der chemischen Analysen die Zellsaftkonzentrationen der nachgewiesenen Ionen berechnet.

4. Die Voraussetzungen für die Zuverlässigkeit der erzielten Ergebnisse werden im Zusammenhang mit Befunden anderer Autoren diskutiert.

Meinen Mitarbeitern, Herrn J. K. Holtmann und Fräulein K. Vogt, danke ich für die Durchführung der chemischen Analysen und für die Anfertigung der Zeichnungen.

\section{ZITIERTE LITERATUR}

BuILER, G. W., 1953. Ion uptake by young wheat plants. 2. The apparant free space of wheat roots. Physiol. Plant. 6, 617-635.

Coldander, R., 1930. Permeabilitätsstudien an Chara ceratophylla. 1. Die normale Zusammensetung des Zellsaftes. Acta bot. fern. 6, 1-20.

- 1939. Permeabilitätsstudien an Charazeen. 3. Die Aufnahme und Abgabe von Kationen. Protoplasma 33, 215-257.

- 1956. Der Ort des Penetrationswiderstandes. In: Handbuch d. Pflanzenphysiologie. Hrsg. von W. Ruhland. Springer, Berlin, Bd. 2, 218-229.

COOPER, W. C., Dorcas, M. J. \& Osterhout, W. J. V., 1929. The penetration of strong electrolytes. J. gen. Physiol. 12, 427-433.

Dainty, J., 1960. Ion transport across plant cell. membranes. Proc. R. Soc. Edinb. (B) 28, $3-14$.

Diamond, J. M. \& Solomon, A. K., 1959. Intracellular potassium compartments in Nitella axilaris. J. gen. Physiol. 42, 1105-1121.

EprLer, R. W., 1958. Sodium exclusion and potassium retention by the red marine alga, Porphyra perforata. J. gen. Physiol. 41, 901-911.

- 1959. Potassium accumulation and sodium efflux by Porphyra perforata tissues in lithium and magnesium sea water. I. gen. Physiol. 43, 29-38.

- 1962. Major cations. In: Physiology and biochemistry of algae. Ed. by R. A. Lewin, Acad. pr., New York, p. 255-266.

- \& Blinks, L. R., 1957. Cell space and apparent free space in the red alga, Porphyra perforata. Plant. Phystol. 32, 63-64.

- \& Cyrus, C. C., 1960. Cation regulation and survival of the red alga, Porphyra perforata, in diluted and concentrated sea water. Biol. Bull. mar. biol. Lab., Woods Hole 118, 55-65.

Follmann, G. \& Follmann-Schrag, I. A., 1959. Die Wasserführung plasmolysierter Protoplasten yon Cladophora gracilis unter dem Einfluß von Atmungsgiften. Z. Naturf. 14 b, $181-187$.

Hormann, C., 1932. Zur Bestimmung des osmotischen Druckes an Meeresalgen. Planta 16, $413-432$.

Holm-Jensen, I., Krogh, A. \& WartiovaAra, V., 1944. Some experiments on the exchange of potassium and sodium between single cells of Characeae and the bathing fluid. Acta bot. fenn, 36, 1-22. 
Hope, A. B., 1953. Salt uptake by root tissue cyptoplasm: The relation between uptake and external concentration. Aust. J. biol. Sci. 6, 396-409.

- \& Stevens, P. G., 1952. Electrical potential differences in bean roots and their relation to salt uptake. Aust. J. sci. ent. Res. (B) 5, 335-343.

Kотте, H., 1914. Turgor und Membranquellung bei Meeresalgen. Wiss. Meeresunters. Abt. Kiel 17, 119-167.

Kramer, P. J., 1956. The uptake of salts by plant cells. In: Handbuch d. Pflanzenphysiologie. Hrsg. von W. Ruhland. Springer, Berlin, Bd. 2, 290-315.

MacRobbie, E. A. C., 1962. Ionic relations of Nitella translucens. J. gen. Physiol. 45, 861-878.

- 1964. Factors affecting the fluxes of potassium and chloride ions in Nitella translucens. J. gen. Physiol. 47, 859-877.

- \& Darnty, J., 1958. Ion transport in Nitellopsis obtusa. J. gen. Physiol. 42, 335-353.

SCOTT, G. T. \& HAYWARD, H. R., 1954. Evidence for the presence of separate mechanisms regulating potassium and sodium distribution in Ulva lactuca. J. gen. Physiol. 37, 601-620. 